EUROPEAN ORGANIZATION FOR NUCLEAR RESEARCH

CERN/PS/LEA 82-14

August 1982

\title{
CONSTRUCTION OF THE LEAR FACILITY: STATUS REPORT
}

P. Lefèvre

Paper presented at the Workshop on physics at LEAR

with low energy cooled antiprotons

Erice (Sicily), May 9-16, 1982 
. 
CONSTRUCTION OF THE LEAR FACILITY: STATUS REPORT

P. Lefèvre

PS Division

CERN, CH-1211 Geneva 23, Switzerland

\section{INTRODUCTION}

The construction of LEAR has now entered into its decisive phase. In this talk I will try to recall the main features of this (small but beautiful) appendix to the large antiproton complex and then give the present status and the expected advancement of the assembly and the running-in. We shall conclude that with some luck, with the hard work of the LEAR team and with the active help of almost all groups and services of PS Division, we shall be able in about one year from now to supply you with antiprotons for the start of the unique physics programme which will be discussed during most of this week.

MAIN MACHINE CHARACTERISTICS

\section{Antiprotons and test particles for LEAR}

The ${ }_{1}$ general antiproton scheme was described in the talk by E. Jones. LEAR is one of the 3 customers of the AA.

For physics runs with LEAR a pulse of $10^{9} \overrightarrow{\mathrm{p}}$ will be taken from the AA once every $10^{3} \mathrm{~s}$, passed through the PS and injected into LEAR at $0.6 \mathrm{GeV} / \mathrm{c}$. In LEAR itself the antiprotons can be accelerated up to $2 \mathrm{GeV} / \mathrm{c}(1.3 \mathrm{GeV}$ kinetic enexgy) or decelerated to $0.1 \mathrm{GeV} / \mathrm{c}$ ( $5 \mathrm{MeV}$ kinetic energy). Both the highest and the lowest energies require efficient field correction, precise beam handling, cooling, etc. - in short a thorough understanding of the machine. It is therefore not reasonable to expect the extreme 
TABLE 1

LOW ENERGY ANTIPROTON RING (LEAR)

\section{GENERAL SCHEME}

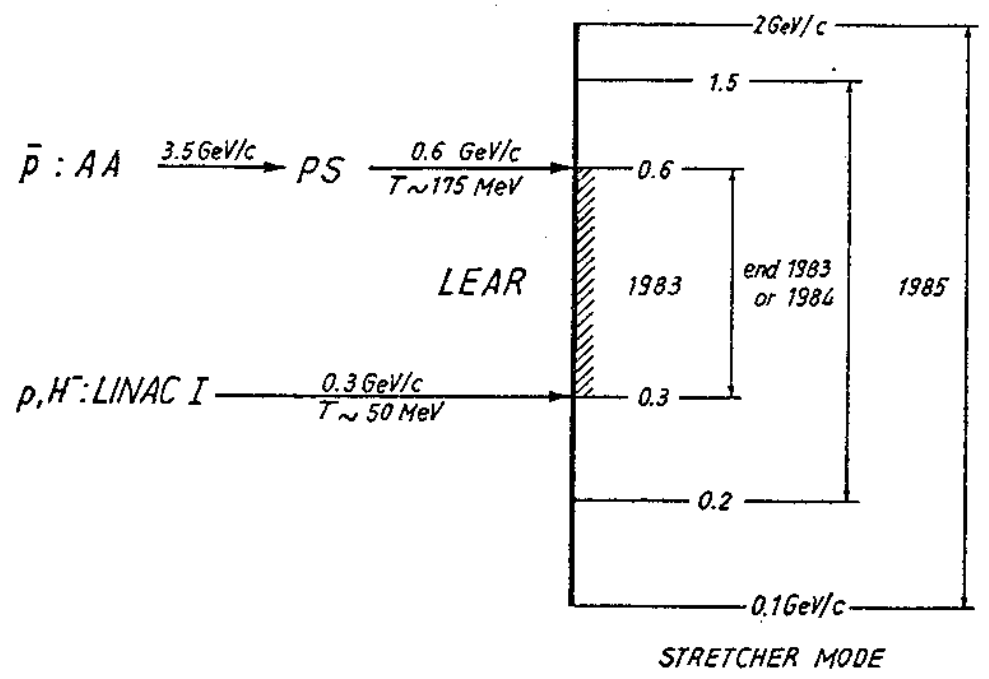

momenta too early in the life of LEAR but at later dates as indicated in Table 1 .

For machine tests, LEAR can work with protons or negative hydrogen ions $\left(\mathrm{H}^{-}\right)$from the old linac which will become an integral part of the LEAR complex. The loop (Fig. 1) which bends the Linac beam back into the LEAR injection line has been installed in the PS tunnel during the last PS annual shutdown.

Test particles from the Linac have to be accepted by LEAR at the linac energy of $50 \mathrm{MeV}(\approx 0.3 \mathrm{GeV} / \mathrm{c})$. $\mathrm{H}^{-}$allow one to test the machine with "p polartiy" in the range of $0.65-0.1 \mathrm{GeV} / \mathrm{c}$ where the $\mathrm{H}^{-}$are stable; but the stripping on the rest gas Iimits the $\mathrm{H}^{-} 1$ ifetime to 20-200 s at the best LEAR vacuum ( $2 \times 10^{-12}$ Torr) and to correspondingly shorter times at higher rest gas pressure. Tests with protons require inversion of the polarities of all fields of the ring and the lines. 


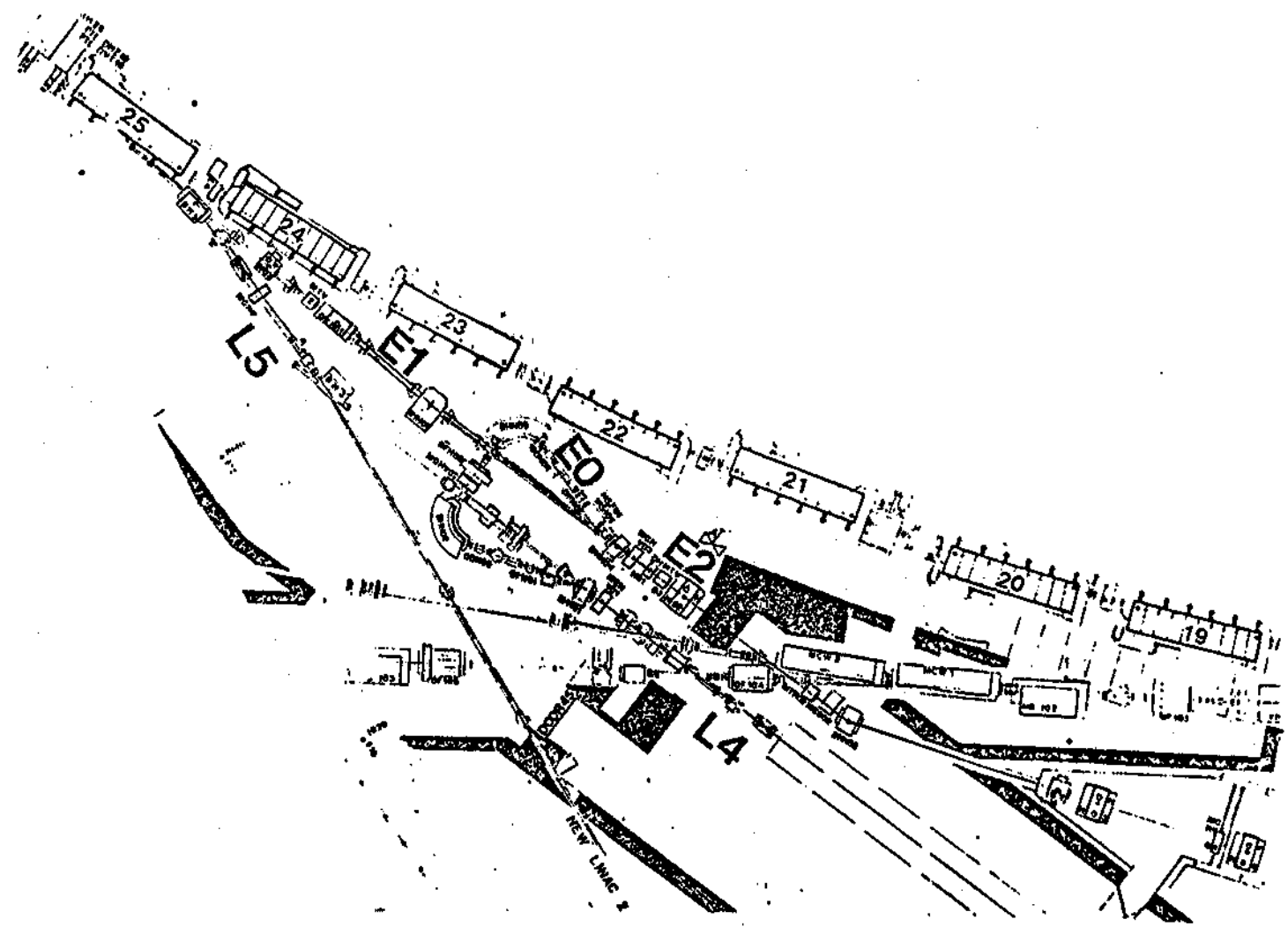

Fig. 1 - Transfer lines PS-LEAR (E1, E2) and Linac1-LEAR (L4, E0)

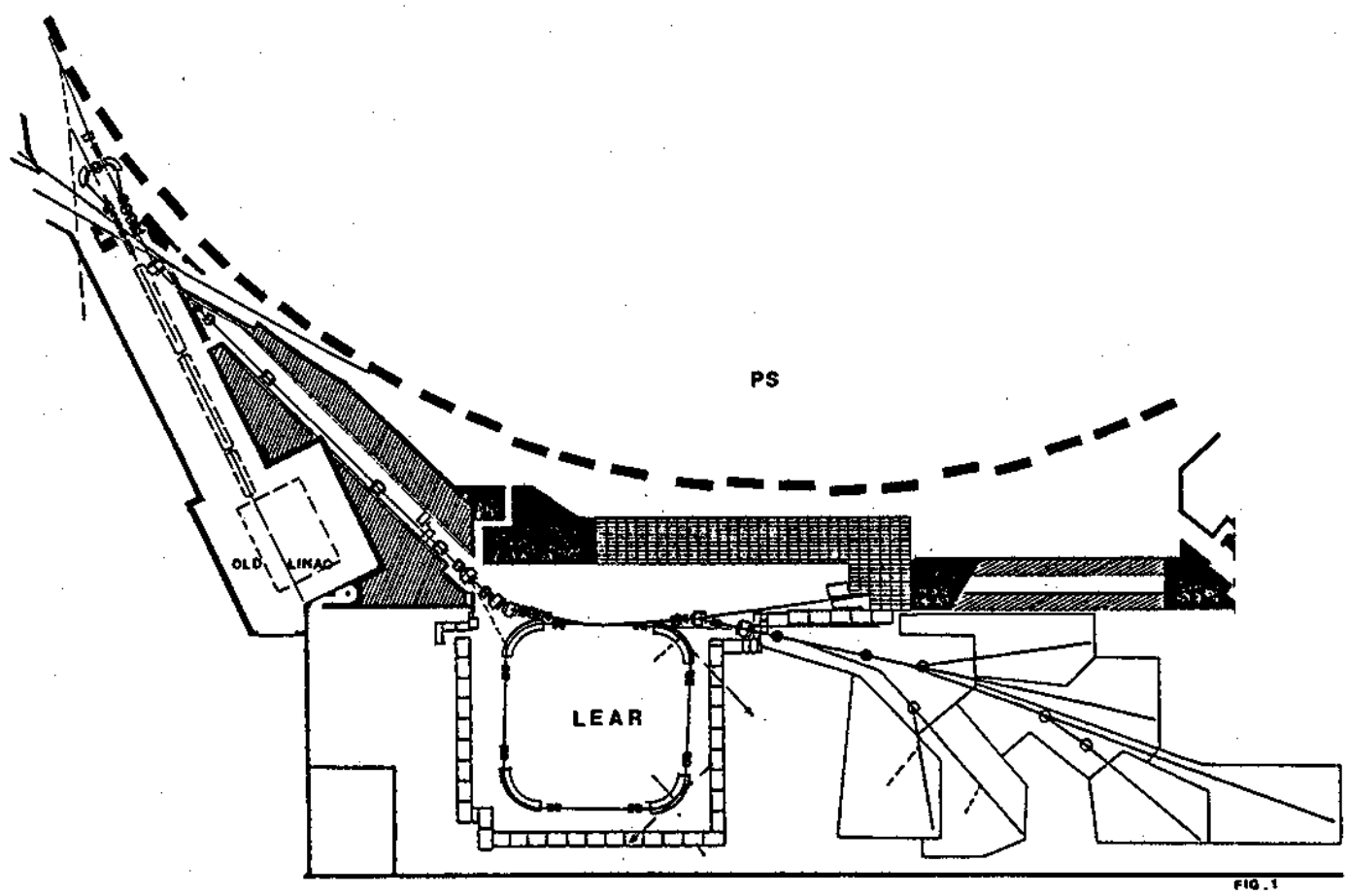

Fig. 2 - LEAR implantation transfer lines - Experimental area. 
During the next years, antiprotons for machine test will only be available on very few occasions. It is therefore very important for a smooth commissioning of LEAR that the linac can work practically in permanence during the rumning-in period. It should also be kept in mind that a sufficient number of development runs with antiprotons is indispensable: due to the fragility of the $\mathrm{H}^{-}$and the problems of polarity inversion with test protons a number of tests can only be performed with $\bar{p}$ themselves.

General layout

The choice to build LEAR in the PS South Hall (Fig. 2) has conditioned many machine parameters such as size (one eighth of the PS) and the geometry of the "ring" and the layout of the ejected beam and the experimental area. This area now extends into the East part of the South Hall (Fig. 2). This new layout, which was recently adopted, permits the simultaneous presence of seven experiments as we will hear in $D$. Simons talk ${ }^{2}$.

The injection line from PS to LEAR is taken through a new piece of tunnel, which was excavated about one year ago. The $\bar{p}$ beam is ejected from the PS straight section 26. The "ejection" septum magnet can also be used for the injection into the PS of protons and ions coming from Linac $I$ or Linac II.

The Linac II control room, extended by a terminal room and an equipment room, will also serve as the control centre for LEAR. Experiments will obtain information and limited control on mobile terminals. Correction element supplies, electronic equipment and supplies for sub-systems (injection, ejection, RF, baking) will be located in the area between LEAR and the Linacs, or in the LEAR machine area. The power supplies for the LEAR magnets and quadrupoles and the beam elements will be installed inside a new South Rectifier Building (SRB2).

LEAR will be enclosed in concrete shielding walls designed to keep the radiation in the PS Main Control Room, which is permanently occupied, at acceptable levels.

\section{Brief description of some components}

A summary of LEAR parameters is given in Table 2 .

The machine is almost square (Fig. 3) in order to provide long straight sectors where large equipment can be installed (e.g. a low-beta section and/or large equipment around an internal target or a collision point). The four sector magnets and a quadrupole doublet at either side of it provide for short straight sections of $1 \mathrm{~m}$ length between dipole and quadrupoles and long straights of $8 \mathrm{~m}$ between the doublets. The beam height is $1.66 \mathrm{~m}$ above the floor of the hall. 


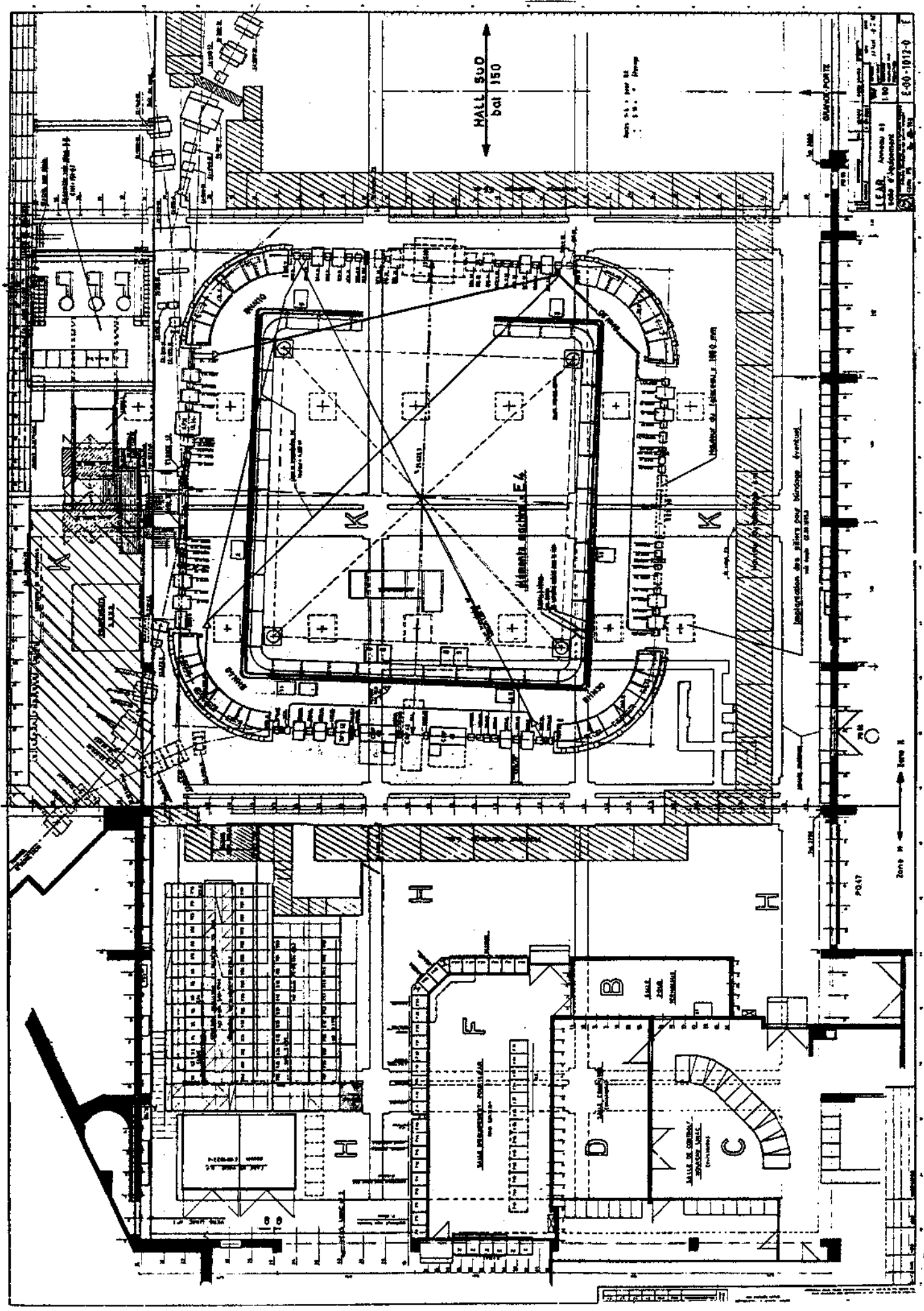

Fig. 3 - The LEAR enclosure (K) and the control (C) and equipment room (E). 
Momentum (kinetic energy) range

Injection momentum (kinetic energy)

Circumference

Typical cycle

Typical extracted beas

Typical spill length

Long straight sections

Short straight sections

(between quadrupoles and Bending Magnet)

Bending magnets, No. arc length, field at $2 \mathrm{GeV} / \mathrm{c}$

Quadrupoles, No., magnetic length, max gxadient

at $2 \mathrm{GeV} / \mathrm{c}$

Pocusing structure

Betatron wave number ${ }^{*}$

Momentum compaction ${ }^{*}$

Aperture limitations

Acceptances ${ }^{*}$ )

Vacuum system design pressure ( $N_{2}$ equivalent)

Bake out termerature

Pump down time

$R F$ system frequency range $(h=1)$

Peak voltage per turn ${ }^{*}$

PS beam bunch properties at transfer ( $h=10$ in PS),

area, total bunch length

Acceptances of transfer system

Assumed AA beam properties at $3.5 \mathrm{GeV} / \mathrm{c}$

(unstacking of $10^{9} \bar{p}$ )

Longitudinal acceptance: PS

LEAR

Longitudinal emittance: PS

\section{IEAR}

\section{$0.1-2 \mathrm{GeV} / \mathrm{c}(5.3 \mathrm{MeV}-1.3 \mathrm{GeV})$ \\ $0.6 \mathrm{GeV} / \mathrm{c} \quad(175.4 \mathrm{MeV})$ \\ $78.54 \mathrm{~m} \quad(=2 \pi \times 12.5 \mathrm{~m})$}

$10^{9} \bar{p}$ injected every $10^{3} \mathrm{~s}$

$10^{6} \overline{\mathrm{p}} \mathrm{p} / \mathrm{s}$

$900 \mathrm{~s}$

4 of 8 m length each

8 of 1 m length each

$4,6.55 \mathrm{~m}, \mathrm{~B}=1.6 \mathrm{~T}$

$16,0.5 \mathrm{~m}, \mathrm{k}=1.8 \mathrm{~m}^{-2}(\mathrm{~g}=12 \mathrm{~T} / \mathrm{m})$

4 superperiods, sepaxated function BODFOFDOB

$Q_{H} \simeq 2.3, Q_{V} \simeq 2.7$

$\alpha=\gamma_{t r}^{-2}=-4.8 \times 10^{-3}$

$a_{H}= \pm 70 \mathrm{~mm}, \quad \alpha_{j}=29$

$\hat{E}_{H}=240 \pi m$ mrad

$\hat{\mathrm{E}}_{\mathrm{V}}=48 \pi \mathrm{mrad}$

$\frac{\Delta p}{p}=1.17$

$10^{-22}-10^{-12}$

$300^{\circ} \mathrm{C}$

$40 \mathrm{~h}$.

$0.4-3.5 \mathrm{MRz}$

$12 \mathrm{kV}$

A $=15$ mrad, $l_{b}=250-300 \mathrm{~ns}$

$\hat{\mathrm{E}}_{\mathrm{H}}=40 \pi \operatorname{marad}$

$\hat{\mathrm{E}}_{\mathrm{V}}=20 \pi \mathrm{mm} \mathrm{mrad}$

$\frac{\Delta p}{p}= \pm 5 \times 10^{-3}$

$\mathrm{E}=3.0 \pi \mathrm{mm} \mathrm{mrad}$

$E_{0}=1.5 \pi \mathrm{mrad}$

$A=6 \operatorname{mrad}(\operatorname{at} h=1$ in $A A)$

$\hat{A}=25$ mrad $(h=10)$

$\hat{A}=20$ arad $(h-1)$

$A=15 \mathrm{mrad}(\mathrm{h}=10)$

$A=12$ mrad $(h-1)$

* Seretcher mode 
The al1-metal vacuum systems will be designed for baking at $300^{\circ}$

with the ultimate goal of achieving the vacuum limits obtained in the ISR. The acceleration system consists of two ferrite cavities.

Controls will be based on the PDP $11 / 45$ computers of the Linac control system, to which a third CAMAC branch is being added.

Injection and ejection make use of a common septum magnet in SL1. The injected beam receives its final deflection by a fast ferrite kicker. Space is reserved in SL4 for a $\mathrm{H}^{-}$charge exchange injection system to get a proton beam, circulating in a direction opposite to that of the antiprotons. The ejected beam is deflected across the common injection-ejection magnetic septum by a thin electrostatic septum device in ss 11.

To obtain a continuous ejected beam between two refills, a special ejection system providing a very long spill was designed. It makes use of the phenomenon that an RF signal, containing noise of a certain bandwidth, induces in the beam a diffusion process inside the corresponding momentum band. This technique is used first to flatten the particle distribution and then to make particles diffuse until, at a momentum corresponding to a transverse resonance, ejection takes place. More details about this ultra slow extraction method (also called stochastic ejection) will be given in a separate talk by R. Cappi, R. Giannini and W. Hardt ${ }^{3}$. We note here that the spill rate of $10^{6} \mathrm{p} / \mathrm{s}$ corresponds to the ejection of about one antiproton per turn.

The installation of systems for stochastic transverse and momentum cooling has been studied and they will be available at the first stage of LEAR for initial cooling at injection energy, as we will hear in the talk by $D$. Möh 1 . Provision has been made to have, if desired, additional stochastic cooling facilities later on. The ICE electron cooling system has been adapted to the specification for LEAR (momenta up to about $0.3 \mathrm{GeV} / \mathrm{c}$, short cooling times) and could become usable in 1984 or 1985 as we will learn from the paper 5 to be presented by H.' Poth.

The further options, like e.g. internal gas jet targets, corotating $\mathrm{H}^{-}$and $\overline{\mathrm{p}}$ beams, and $\mathrm{p} \overline{\mathrm{p}}$ collision experiments, impose stringent requirements on the machine in order to reach full potential. Up to the present, parameters and choices have been avoided which would exclude the implementation of these options. In defining equipment and dimensions an attempt has been made to give the maximum freedom for these future developments. Examples are the choice to have the yoke on the inside of the magnet, to leave a gap in the centre of the magnet and to equip the vacuum chamber with exit "stubs" for neutrals created in the straight section. Further, space for experimental apparatus is reserved in straight section 2 and the inclusion of a low beta optics in this section is prepared. 
The Antiproton Accumulator is designed to accumulate $6.10^{11} \overline{\mathrm{p}}$ per day when the PS runs for $\overline{\mathrm{P}}$ production exclusively and works at about $1 / 5$ of this at present, as we have heard in E. Jones' talk. Taking into account transfer losses, 4 to $5.10^{6} \overline{\mathrm{p}} / \mathrm{s}$ will be the maximum flux obtainable in the external beam from LEAR when the AA works according to design specifications. It is unlikely that LEAR will be the only facility in operation and the possibility of running in parallel with PS and SPS physics has been considered since the early studies of LEAR.

Two basic schemes of beam sharing are possible:

i) sharing protons, i.e. using one or two $25 \mathrm{GeV} / \mathrm{c}$ cycles within a PS/SPS supercycle for $\bar{p}$ production, the AA being used for LEAR exclusively ("dedicated mode");

ii) sharing antiprotons, i.e. taking a small fraction of a $\bar{p}$ stack being prepared in the AA for the SPS or ISR ("parasitic mode").

The first of these possibilities looks quite straightforward. It would take one or two hours to create in the AA a stack of about $5.10^{10} \overline{\mathrm{p}}$; and unstack batches of the order of $10^{9} \overline{\mathrm{p}}$ at intervals of $1000 \mathrm{~s}$ which, after transfer to LEAR, would be available as an extracted beam with a flux of $10^{6} \overline{\mathrm{p}} / \mathrm{s}$. The batch size of $10^{9} \overline{\mathrm{p}}$ is the minimum which the present PS beam control system needs to function safely.

The second possibility, parasitic running during sPS $\bar{p}$ experiments, would be very interesting if, say, 5 to $10 \%$ of the stack could be allowed for low energy $\bar{p}$ physics. LEAR could then typically receive 30 batches of $10^{9} \overline{\mathrm{p}}$ and run about eight hours per day as a rule. If the PS beam control, on the other hand, could be made more sensitive, so allowing smaller batches to be transferred, or if the slow extraction of LEAR could be stretched out by another factor of three, a parasitic beam of 2 to $3.10^{5} \overline{\mathrm{p}} / \mathrm{s}$ would be available around the clock. The problems associated with the parasitic mode are not yet fully solved. However, recently, a new method of unstacking sam11 batches from the AA has been successfully experimented as we have heard in $\mathrm{E}$. Jones' talk'.

PRESENT STATUS AND FUTURE PROSPECTS

A view of the machine as of May 20, 1982 is given by the photograph (Fig. 4). 


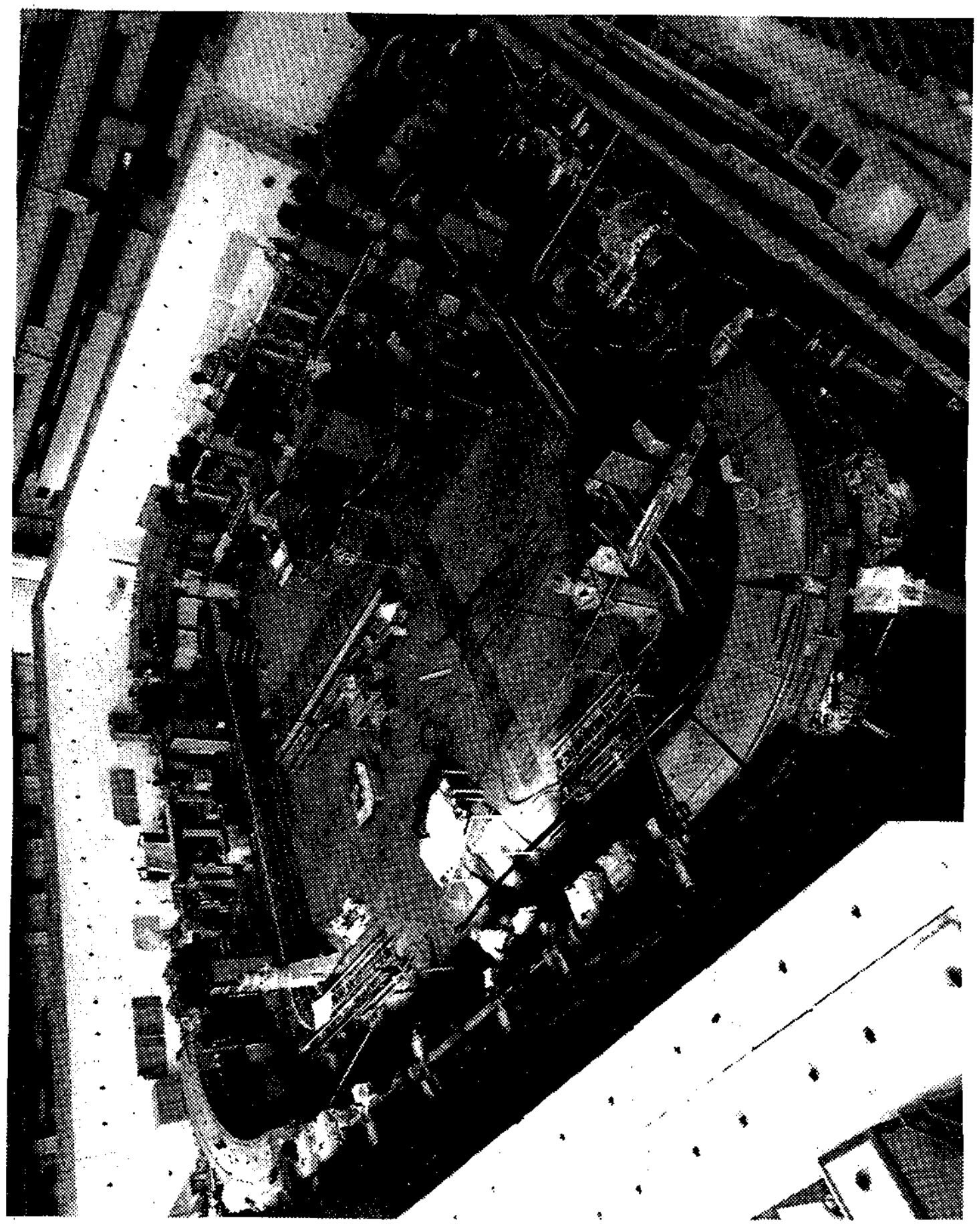

Fig. 4 - General view of the LEAR machine 
Beam Qualities and Acceptances during Transfer

\begin{tabular}{|c|c|c|c|c|c|c|c|c|}
\hline \multirow[b]{3}{*}{$\begin{array}{l}\text { AA Enittances } \\
\text { Partial cooling } \\
\text { Total cooling }\end{array}$} & \multirow{3}{*}{$\begin{array}{c}\mathrm{cp} \\
\mathrm{GeV} \\
3.5 \\
\end{array}$} & \multicolumn{4}{|c|}{ Fhysical enittances } & \multicolumn{3}{|c|}{ Normalized emittances } \\
\hline & & $\varepsilon_{H}$ & $\begin{array}{l}\varepsilon_{V} \\
\text { 1.mrad }\end{array}$ & $\begin{array}{c}\frac{\Delta P}{p} \\
\pm 10^{-3}\end{array}$ & A & $\varepsilon_{H}^{\pi}$ & $\varepsilon_{V}^{*}$ & $\begin{array}{c}A^{*} \\
e V / s\end{array}$ \\
\hline & & $\begin{array}{l}4.5 \\
2.1\end{array}$ & $\begin{array}{l}2.2 \\
1.1\end{array}$ & & $6=\frac{72}{12}$ & $\begin{array}{r}17 \\
8\end{array}$ & $\begin{array}{l}8.4 \\
4\end{array}$ & 0,47 \\
\hline $\begin{array}{l}\text { Acceptances } \\
\text { PS Ejection } \\
\text { PS transfer } \\
\text { LEAR injection } \\
\text { LEAR ring }\end{array}$ & 0.6 & $\begin{array}{r}40 \\
40 \\
40 \\
240\end{array}$ & $\begin{array}{l}20 \\
20 \\
20 \\
48\end{array}$ & $\begin{array}{r}5 \\
5 \\
5 \\
11\end{array}$ & $\begin{array}{l}25 \\
20\end{array}$ & 28 & 14 & 0.78 \\
\hline $\begin{array}{l}\text { LEAR injection } \\
\text { expeced } \\
\text { emittances }\end{array}$ & 0.6 & 30 & 15 & 3 & $12-15$ & 21 & 10.5 & $0.5-0.6$ \\
\hline
\end{tabular}

Expected Beam Performances at LEAR

EXPECTED BEAM PERFORMANCES AT LEAR

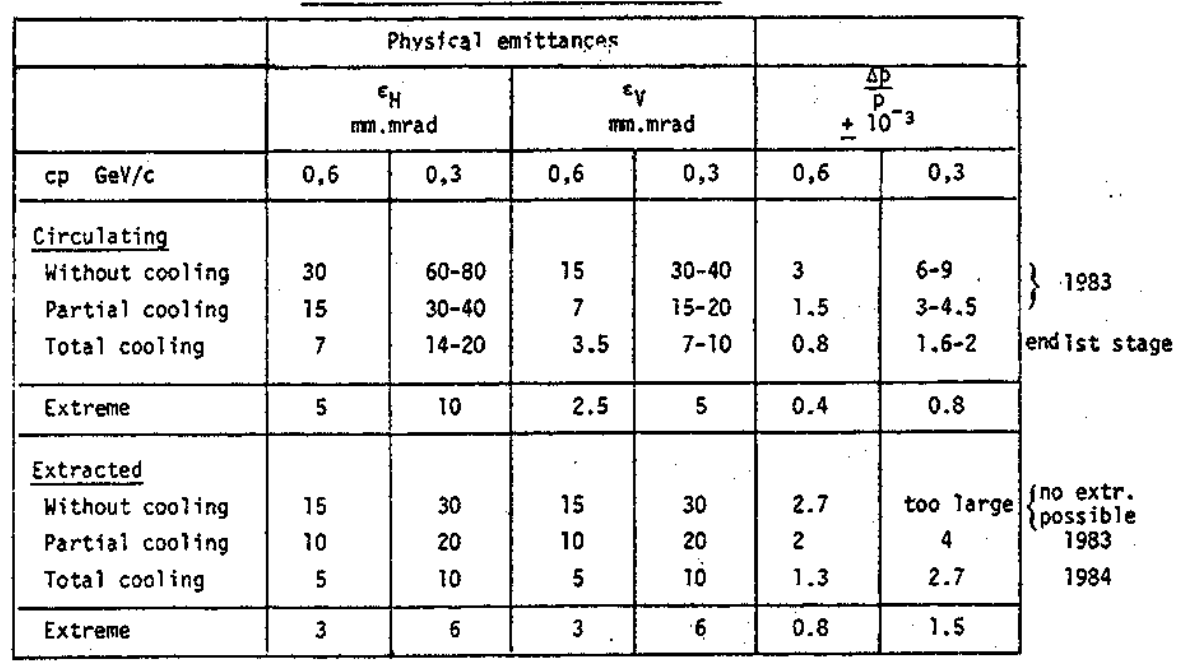

Partial cooling: 3 " with intial stochastic cooling Total cooling : 2' with initial stochastic cooling Extreme: reduce $A A$ enittances

PA-PS-LEAR

improve AA cooling (S, van der Meer system)

increase LEAR cooling efficiency (reduced pick-up signai)

Units (95\%) (1.5 2o emit. for Gaussian distribution)

$$
\begin{aligned}
& \varepsilon_{4}, \varepsilon_{V}=\text { surface } / \pi \quad \varepsilon^{*}=\text { BY } \varepsilon \\
& \left.\begin{array}{lrl}
A \text { in }\left(\frac{\Delta p}{m_{0} c}, \Delta \phi_{R F}\right) & A=\begin{array}{r}
6 \mathrm{mrad} \\
15 \mathrm{mrad}
\end{array} \\
A^{*} \text { in }(\Delta E, \Delta t) & \begin{array}{l}
\text { PA } \\
\text { LEAR }
\end{array}
\end{array}\right\}=0.47 \mathrm{mrad}=A^{*}
\end{aligned}
$$


The installation is progressing at full speed. About one third

of the components are "in lieu". The vacuum system will be closed in July, so that the first injection test can hopefully start before August. We hope that by December the running-in with test particles is sufficiently advanced so that a dedicated $\bar{p}$ run of a few days becomes meaningful. Such a test with antiprotons, under almost operational conditions is essential to establish the complicated succession of operations in AA, PS and LEAR necessary to supply the users community reliably with a high quality beam. A second $p$ test run in 1983 after the annual shutdown may be necessary for final debugging .

It is clear that there are uncertainties as with any new machine of the complexity of LEAR. But we will work hard to give you protons for test purposes into the experimental areas in April next year and first antiprotons in May. Following your suggestions, we will try to start immediately with antiprotons decelerated to $0.3 \mathrm{GeV} / \mathrm{c}$ although operation at $0.6 \mathrm{GeV} / \mathrm{c}$ would have been easier for us.

\section{EXPECTED BEAM PROPERTIES FOR INITIAL OPERATION}

Table 3 tries to give an idea of the beam performances which we hope to achieve in the initial operation and later on. It is clear that a "better" beam can be supplied if you do not ask for too frequent changes and exotic operation during the first period of runs when LEAR will be liable to all sorts of childhood diseases. As an example, any breakdown which needs opening of the vacuum system will cost a minimum of $48 \mathrm{~h}$ of bake out and pump down, apart from the time for diagnostic repair and restart.

This being said, we will try hard to provide you with a good antiproton beam for first generation experiments as early as possible, which, due to the overall schedule, means May 1983. And we hope to improve the quality of this beam so that even the most demanding experiments can be satisfied in 1984/85 and beyond.

\section{References}

1. E. Jones, The CERN $\overline{\mathrm{p}}$ complex and present status and future developments of the Antiproton Accumulator, This Conference.

2. D.J. Simon, The LEAR experimental areas, This Conference.

3. R. Cappi, R. Giannini and W. Hardt, U1traslow extraction (status report), This Conference.

4. D. Möh1, Phase-space cooling techniques and their combination, This Conference.

5. H. Haseroth, C. Hill, L. Hütten, H. Poth and A. Wolf, The electron cooling device for LEAR, This Conference. 
